\title{
Magnetic resonance imaging in prostate cancer
}

Prostate cancer screening and detection has historically been performed through the use of prostate-specific antigen (PSA) and systematic transrectal ultrasound guided biopsy among all men with perceived elevation of PSA level. This diagnostic model has fueled much of the ongoing criticism of prostate cancer screening by contributing to over-diagnosis through random detection of indolent cancer, repetitive biopsy due to concerns of missed high grade tumors, and over-treatment due to a lack of confidence in the accuracy of biopsy risk assessment. Prior to the advent of prostate magnetic resonance imaging (MRI), accurately assessing for the presence and location of clinically significant lesions has been challenging. MRI offers increasingly reliable visualization of potentially significant prostate cancers, thereby guiding biopsy to allow better sensitivity and negative predictive value. In applying MRI-targeted biopsy, we have learned the significance of MRI findings and evolved pre-biopsy MRI to serve as a means by which to better select patients for biopsy and identify lesions for biopsy. Rather than diagnosing all cancers of the prostate, we have now begun the shift the paradigm to selective identification of only those cancers that may cause harm.

As Guest Editors of this issue of TAU, we aimed to provide an in-depth overview of the technology of prostate MRI, the Prostate Imaging Reporting and Data System (PI-RADS), and the role of MRI in targeted prostate biopsy, along with its current and potential clinical applications.

In the pre-biopsy setting, MRI has evolved into a non-invasive tool for prostate cancer risk-stratification that can influence the decision whether to perform biopsy based on MRI suspicion thresholds for biopsy, individualized to the clinical setting. To increase the quality and diagnostic value of prostate MRI, the International Prostate MRI Working Group developed PIRADS. Major goals of the PI-RADS classification are to allow comparison of inter-observer interpretation variability; to enhance communication with clinicians in a uniform way; to facilitate quality assurance and research; and to improve patient outcome. Using PI-RADS, the likelihood of clinically significant cancer is correlated with increasing suspicion on MRI.

In addition to risk assessment prior to biopsy, the ability to distinguish between organ-confined tumors $(\leq \mathrm{T} 2 \mathrm{c})$ and those that extend beyond the prostate $(\geq \mathrm{T} 3 \mathrm{a})$ is an important component of decision-making prior to treatment. Through the emergence of multiparametric imaging, MRI has become more useful for predicting extraprostatic extension at surgery and may play an increasing role in surgical planning.

In men presenting for first biopsy, the potential advantages of MRI are twofold: improving detection of high-grade cancer by reducing the false-negative rate of biopsy, and avoiding detection of low-grade disease by selectively targeting tumor foci which are likely to be clinically significant. Among men with a prior negative biopsy, the advantage of MRI and targeted biopsy is in identifying areas of suspicion within the prostate that otherwise would be missed by repeat systematic sampling. Prostate MRI may have two applications in men with a prior positive prostate biopsy. First, it improves risk stratification for selecting appropriate candidates for active surveillance (AS) by allowing the identification and targeting of MRI suspicious regions with biopsy, ultimately ruling out clinically significant disease. Secondly, it reduces the need for repetitive biopsy through non-invasive serial monitoring for those on AS, due to greater confidence in the biopsy outcome.

Prostate MRI has resulted in a paradigm shift in the way we think about prostate cancer detection, risk assessment, and therapy. Increased experience with MRI and targeted biopsy will broaden our application and deepen our understanding. We are indebted to the world experts in prostate cancer imaging and diagnosis who took time away from their busy clinical practices to contribute these insightful articles.

\section{Acknowledgements}

This work was supported by the Joseph and Diane Steinberg Charitable Trust. 


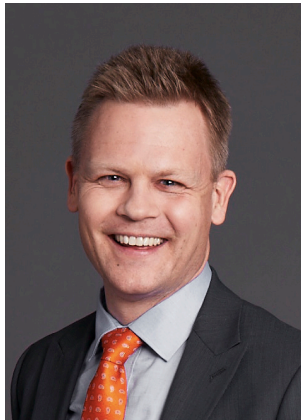

Marc A. Bjurlin

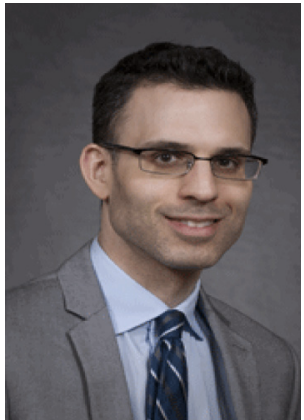

Andrew B. Rosenkrantz

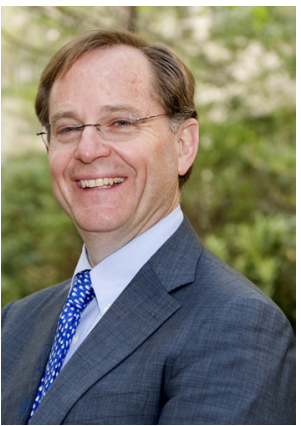

Herbert Lepor

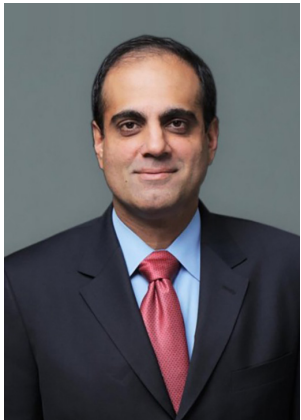

Samir S. Taneja

Marc A. Bjurlin, DO, MSc

Clinical Assistant Professor of Urology; Director of Urologic Oncology; NYU Lutheran Medical Center, New York, NY, USA; Department of Urology, NYU

Langone Medical Center, New York, NY, USA. (Email: Marc.Bjurlin@nyumc.org)

Andrew B. Rosenkrantz, MD

Associate Professor of Radiology and Urology; Director of Health Policy; Director of Prostate Imaging, NYU Langone Medical Center, New York, NY, USA.

(Email: andrew.rosenkrantz@nyumc.org)

Herbert Lepor, MD

Professor and Martin Spatz Chairman of Urology, New York University School of Medicine; Urologist in Chief, NYU Langone Medical Center,

New York, NY, USA.

(Email: Herbert.lepor@nyumc.org)

Samir S. Taneja, MD

The fames M. Neissa and Fanet Riba Neissa Professor of Urologic Oncology; Professor of Urology and Radiology;

Director, Division of Urologic Oncology, NYU Langone Medical Center, New York, NY, USA.

(Email: Samir:Taneja@nyumc.org)

doi: 10.21037/tau.2017.05.16

Conflicts of Interest: Dr. Lepor: MedReviews—Co-owner; Allergan—Speaker Bureau; Serenity—Investor; Thera Coat—Consultant;

Sonacare-Investor; Biozeus_Consultant. The other authors have no conflicts of interest to declare.

View this article at: http://dx.doi.org/10.21037/tau.2017.05.16

Cite this article as: Bjurlin MA, Rosenkrantz AB, Lepor H, Taneja SS. Magnetic resonance imaging in prostate cancer. Transl Androl Urol 2017;6(3):343-344. doi: 10.21037/ tau.2017.05.16 